\title{
A possible 38 day X-ray period of KS 1731-260
}

\author{
M. Revnivtsev ${ }^{1,2}$ and R. Sunyaev ${ }^{2,1}$ \\ 1 Space Research Institute, Russian Academy of Sciences, Profsoyuznaya 84/32, 117810 Moscow, Russia, \\ 2 Max-Planck-Institute für Astrophysik, Karl-Schwarzschild-Str. 1, 85740 Garching bei München, Germany
}

Received 21 August 2002 / Accepted 5 December 2002

\begin{abstract}
We report the detection of a 38 day period of the X-ray transient and X-ray burster KS 1731-260. The periodicity was detected during Mar.1996-Aug.1998 ( TJD 10150-11050) when the source had a high and relatively stable X-ray flux. After Sep.-Oct. 1998 ( TJD 11100) the source became strongly variable on a time scale of months that contaminates the search for the 38 day periodicity. The detected period can not be a binary period. A binary with Roche lobe overflow has in this case large radii of the secondary and of the accretion disk. Illumination of the disk and secondary star by the X-ray flux from the luminous neutron star and inner disk, would lead to a high infrared brightness of the binary. That clearly contradicts the infrared data even for the brightest infrared sources within CHANDRA error box of KS 1731-260. A remaining possibility is that observed periodicity is connected with the accretion disk precession, similar to that was observed for, e.g. SS 433 and Her X-1.
\end{abstract}

Key words. accretion, accretion disks - stars: individual: KS1731-260 - stars: binaries: general - stars: neutron X-ray: stars

\section{Introduction}

KS 1731-260 was discovered in 1989 by the TTM coded mask telescope aboard the MIR-KVANT observatory (Sunyaev 1989; Sunyaev et al. 1990). The archived observations of the TTM telescope showed that the source was not active more than a year before that time with upper limits $\sim 20 \mathrm{mCrab}$ (see Aleksandrovich et al. 2002). Since its discovery the source was extensively observed by various X-ray observatories (e.g. Barret et al. 1993; Barret et al. 1998; Smith et al. 1997; Narita et al. 2001).

In 1997 nearly coherent oscillations were found in the power spectra of this source during a type I X-ray burst (Smith et al. 1997) with a frequency $f \sim 524 \mathrm{~Hz}$ (see Muno et al. 2000 for a review). It is believed that the compact object in the system KS 1731-260 is one of the most rapidly (with spin period $p \approx 1 / f \approx 1.9 \mathrm{msec}$ ) rotating neutron stars. The distance of the source of $d \sim 7 \mathrm{kpc}$ was estimated based on the analysis of the type I X-ray bursts with photospheric radius expansion (Muno et al. 2000).

The source is located close to the Galactic plane and therefore is highly reddened. The recent precise localization of the source with the Chandra observatory strongly increased the possibility that the real infrared counterpart of KS 1731-260 will be found soon (see Barret et al. 1998; Wijnands et al. 2001b; Revnivtsev \& Sunyaev 2002).

In this Letter we present the analysis of the All Sky Monitor (ASM) data aboard the Rossi X-ray Timing Explorer (RXTE)

Send offprint requests to: M. Revnivtsev, e-mail: revnivtsev@hea.iki.rssi.ru and show the presence of a statistically significant periodicity in the X-ray flux of KS 1731-260.

\section{Data analysis and results}

The ASM (Levine et al. 1996) has an intrinsic angular resolution of a few arcminutes, which permits to resolve bright objects even in the very crowded Galactic center region. The ASM provides quite frequent data sampling ( $\sim-15$ times a day, excluding time periods, when the sun inhibits the observations of the Galactic Center region) in three broad energy channels - 1.5-3.0 keV, 3-5 keV and 5-12 keV. KS 1731-260 was monitored by ASM since Feb. 1996.

The light curve of the source obtained by the RXTE/ASM (1996-2001) is presented in Fig. 1 (see also Wijnands et al. 2001a; Aleksandrovich et al. 2002). The flux history of KS 1731-260 could be naturally subdivided into two main parts:

a) Mar. 1996-Aug. 1998 ( TJD 10150-11050), when the source had a relatively stable and high flux;

b) Sep. 1998-Mar. 2001 ( TJD 11100-12000) when strong variability appeared on a monthly time scale.

We searched for periodicities using the Lomb-Scargle periodograms method (Lomb 1976; Scargle 1982; Press et al. 1992) using the light curves of the source in the whole energy band of the ASM (1.5-12 keV). The method of Lomb-Scarge periodograms is the most suitable for the ASM data, because they are distributed non evenly over the total time of the observations. Due to the dependence of the real periodicity peak on 


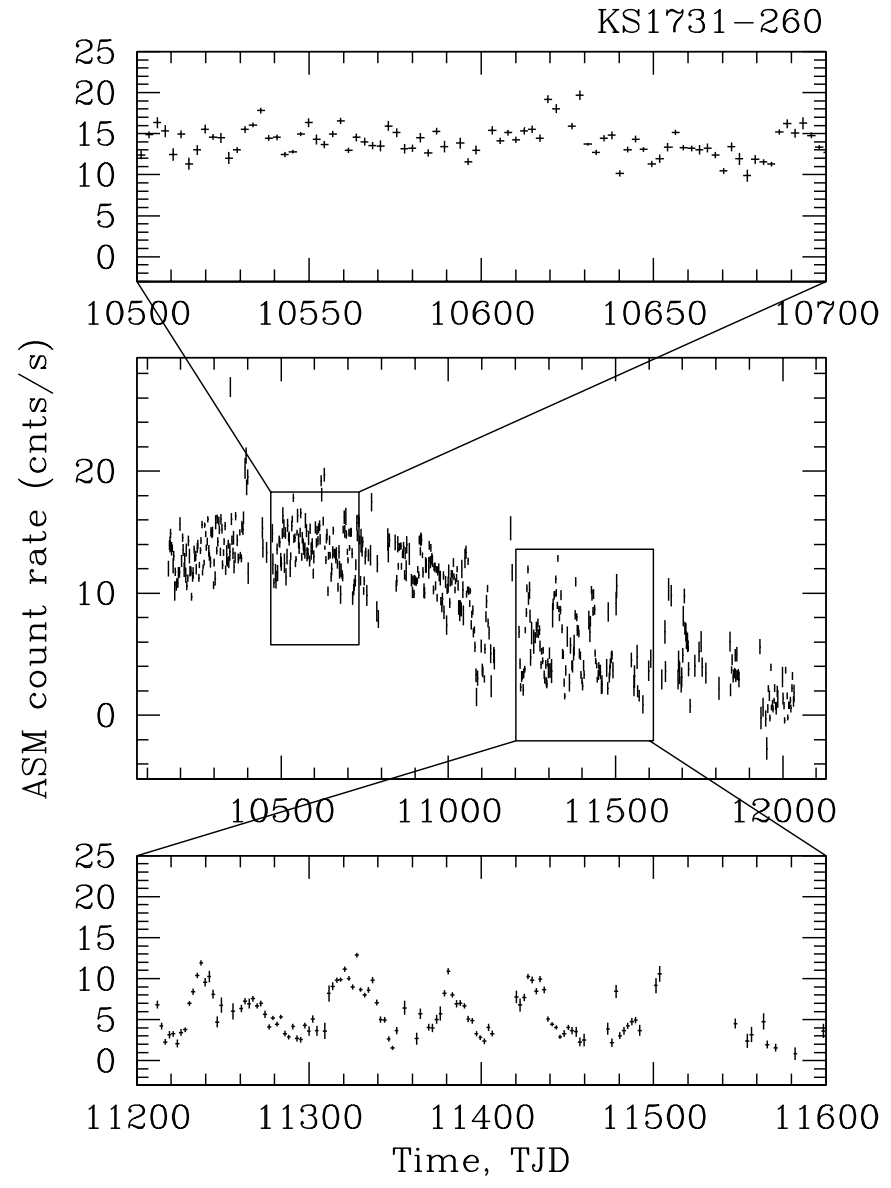

Fig. 1. RXTE/ASM light curve of KS 1731-260 in 1996-2001. The data are binned into 2.5-day bins. Solid lines above the lightcurve denotes approximately the time periods when the periodicity and broad noise were observed. The upper and lower panels show more detailed view of the lightcurve of KS 1731-260 during the periods relatively high flux and period of strong low frequency noise correspondingly.

the number of points used in the Lomb-Scargle periodogram evaluation, for this analysis we used lightcurves of the source with several different binning, from $t_{\text {bin }} \sim 10$ hours to 3 days. The typical Lomb-Scargle periodograms for two abovementioned parts of the flux history of KS 1731-260 are presented in Fig. 2 (for this figure the time binning of the original light curves was chosen to be $\sim 6$ days as a compromise to provide several points per period with highest possible statistics).

a) For the first part of the lightcurve there exist a narrow, prominent and statistically significant $(>6 \sigma)$ peak of the power spectrum of KS 1731-260 at a frequency, corresponding to a period $P \sim 37.67$ days.

We estimated the uncertainty of the period value, using the Monte-Carlo bootstrap method, assuming the Gaussian noise of the initial light curve. The statistical uncertainty of the period value is $\sim 0.03$ days $(P=37.67 \pm 0.03$ days $)$. In order to obtain an additional proof of the presence of the real periodicity in the ASM signal, we made the simple Fourier power spectrum of the highly binned ASM lightcurve (see Fig. 3), and also made the Lomb-Scargle periodograms for all three energy channels of the ASM instrument. The resulted power spectra are presented in Fig. 4.
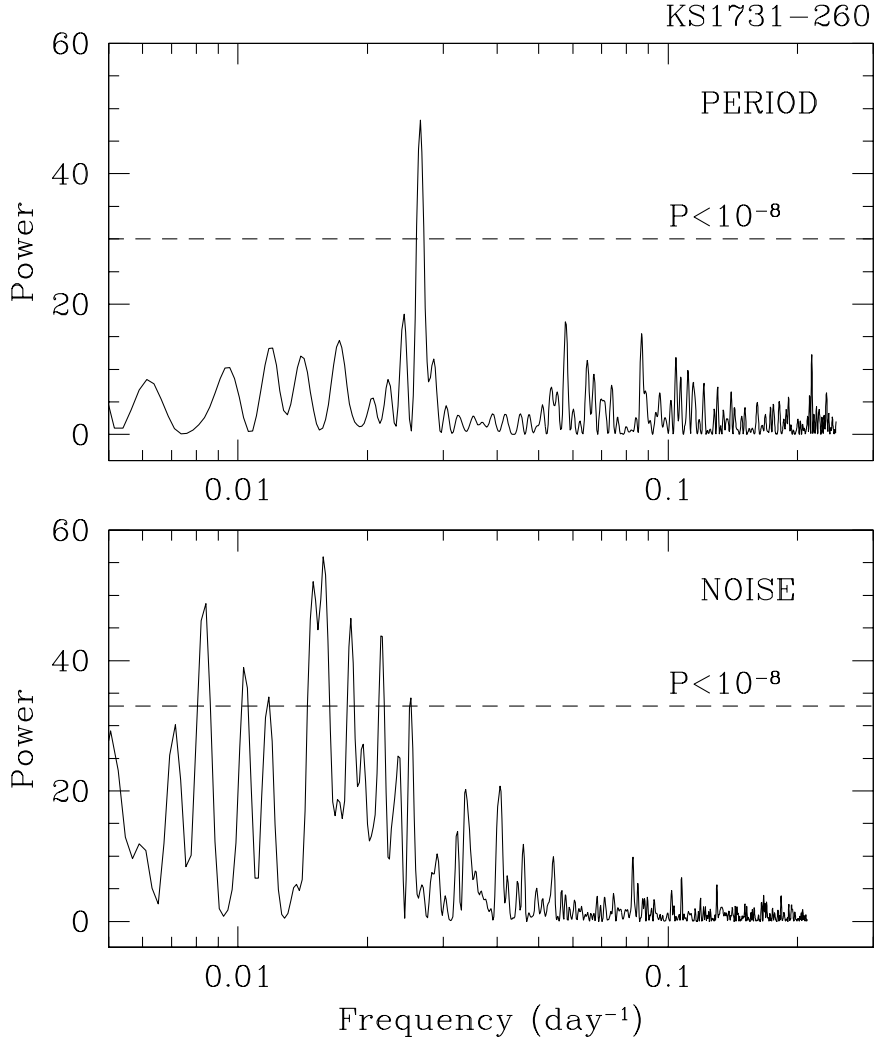

Fig. 2. The Lomb-Scargle periodogram of the light curve of KS 1731-260. The upper panel represents the periodogram made of points $\sim$ Mar. 1996-Aug. 1998, the lower panel $-\sim$ Sep. 1998 Mar. 2001. The dashed line represents the $10^{-8}$ confidence level for the power values, assuming the exponential distribution of noise powers with observed average value (the number of trial points is taken into account).

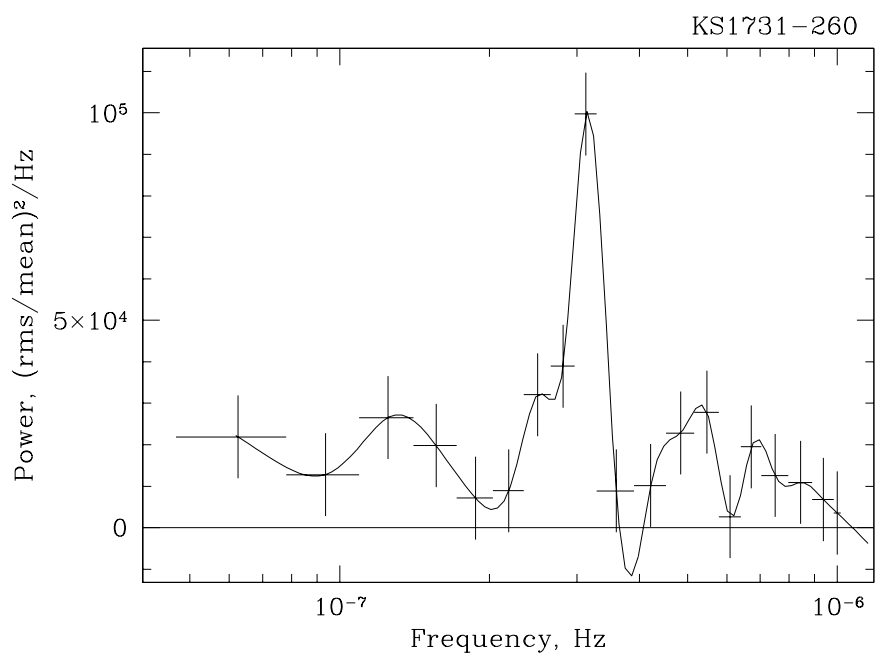

Fig. 3. The power spectrum of KS 1731-260 according to RXTE/ASM data. Original light curve was binned in 4-days bins. The solid line is simple spline to the obtained points.

Then we folded the light curve of KS 1731-260 with the best-fit period of 37.67 days. The resulted phase-intensity diagram of the source is presented in Fig. 5. The folded curve could be well fitted by a sinusoidal wave with amplitude $6 \pm 1 \%$. 

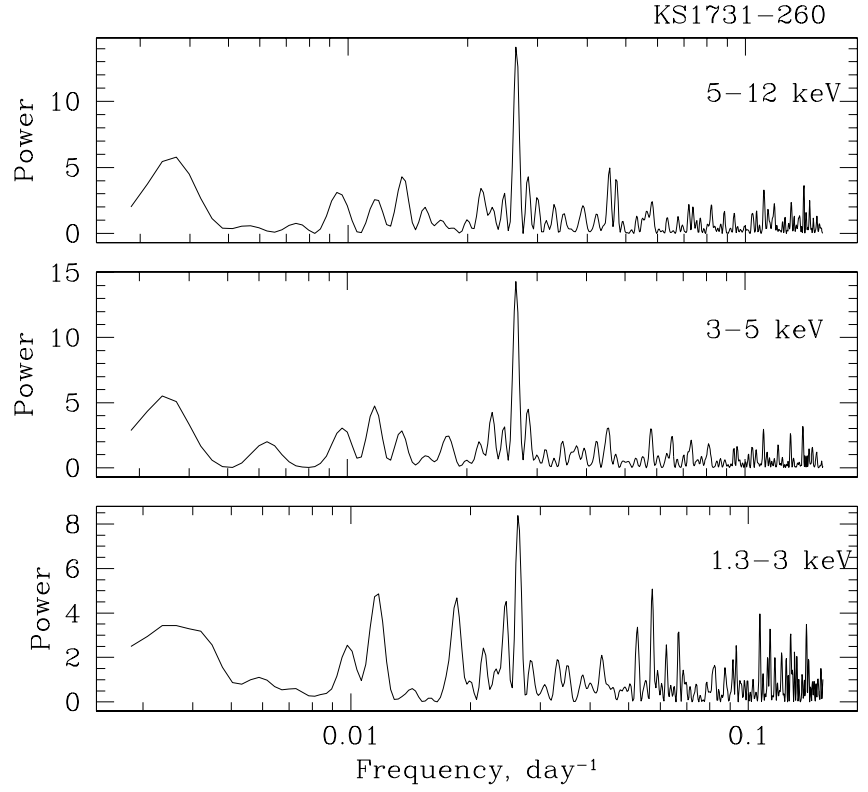

Fig. 4. Three Lomb-Scargle periodograms for three independent ASM energy channels , $1.5-3 \mathrm{keV}, 3-5 \mathrm{keV}$ and $5-12 \mathrm{keV}$.

The amplitudes of the variations in three energy channels are compatible $(6.5 \pm 1.1 \%, 5.8 \pm 0.7 \%, 6.3 \pm 0.7 \%$ for energy channels $1.3-3.0 \mathrm{keV}, 3-5 \mathrm{keV}$ and 5-122 keV respectively). The best fit epoch for the minimum X-ray flux $\left(T_{0}\right)$ is: $T_{0}=10149.05 \pm 1.02$ TJD. The persistence and coherence of the detected variations were tested on the subdivided ASM lightcurves. But, as far as the time interval of our analysis is not very large for our period ( $\sim 24$ periods over $\sim 2.5$ years), we used only two smaller intervals, approximately 450 days each. The best fit amplitudes and the phases of the sinusoidal waves on the folded light curve for each interval are compatible. Therefore it seems that the periodicity in the X-ray flux of KS 1731-260 does not belong preferentially to some particular energy channel of ASM.

b) During the second segment of the lightcurve of KS 1731-260 a set of statistically significant peaks are visible at frequencies 0.01-0.03 day $^{-1}$ (see Fig. 2, lower panel). This peaks presumably represents a broad low frequency noise, and the spiky structure of the power spectrum at that grequency region just reflects tha fact that not all of shown frequency bins are independent. The narrow peak at $\sim 38$ day is not visible anymore. However, it should be noted here that numerical simulations showed that the upper limit of the amplitude of the possible 38 -day variability is not very stringent $<10-15 \%$. This limit is sufficiently higher than the amplitude of the 38 day variations, detected during the first period (see Fig. 5).

\section{Discussion}

Three types of periodicity are known in X-ray binaries: 1) the period of the neutron star rotation, ranging from milliseconds to hundreds of seconds; 2) the orbital period of the binary system, from tens of minutes to tens of days; and 3) the period of the accretion disk precession, as it was observed, e.g. in the case of SS433, Her X-1 etc.

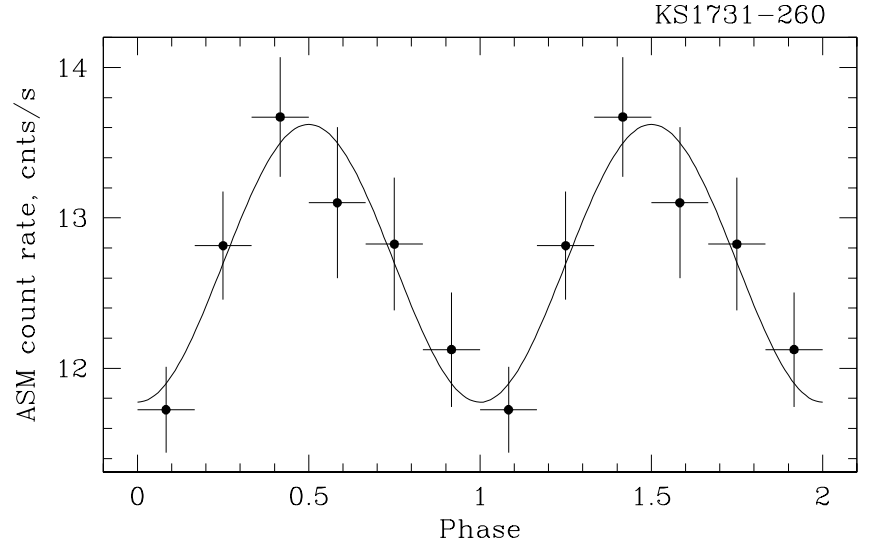

Fig. 5. The folded lightcurve of KS 1731-260. The error bars represents the rms deviations of the points within phase intervals (i.e. not statistical uncertainties).

Observations of nearly coherent oscillations during type I X-ray bursts from KS 1731-260 strongly suggest that the period of a neutron star rotation in this system is close to $1.9 \mathrm{msec}$ (Smith et al. 1997; Muno et al. 2000).

\subsection{Binary period?}

Let us assume that the binary period of KS 1731-260 is $\sim 38$ days and the accretion of the binary system goes due to Roche lobe overflow. The value of the period in this case gives us the binary system separation: $a \sim 3.69 \times 10^{12}(1+q)^{1 / 3} \mathrm{~cm}$ (assuming the mass of the primary $M_{\mathrm{NS}}=1.4 M_{\odot}$ ), where $q-$ is the mass ratio of the companions (see e.g. Warner 1995). The star should have a radius $R_{2} \sim 3.69 \times 10^{12}(1+q)^{1 / 3}(0.38+$ $0.2 \log q$ ) $\mathrm{cm}$ (assuming that $0.3<q<20$ ). Taking $q>0.3$, the minimal possible radius of the secondary could be estimated to be $R_{2} \gtrsim 15 R_{\odot}$.

Now, using this minimal radius of the secondary and the source distance $d \sim 7 \mathrm{kpc}$ we can calculate the minimal apparent infrared magnitudes of the binary for any assumed temperatures of the secondary star surface. These estimates are very important in the view of the progress, that was achieved recently in the search of the KS 1731-260 infrared counterpart (see e.g. Barret et al. 1998; Wijnands et al. 2001b; Revnivtsev \& Sunyaev 2002; Wijnands, private communication). The object, that is claimed to be infrared counterpart of KS 1731-260 had an apparent magnitude $m_{J}>17$ during the infrared observations of Barret et al. 1998. These observations took place when KS 1731-260 was luminous in X-rays $\left(L_{\mathrm{x}} \sim 3 \times 10^{37} \mathrm{erg} / \mathrm{s}\right)$. The limit $m_{J} \gtrsim 17$ could only be satisfied if the $15 R_{\odot}$-star surface temperature is less than $T \sim 1600 \mathrm{~K}$ (the boundaries of the $J$ band and the reference flux were taken from Zombeck 1990). The extinctions $A_{J} \sim 2$ and $A_{H} \sim 1.25$ (see Barret et al. 1998) were taken into account when we were making these estimates. Recent infrared observations suggest that the infrared counterpart of KS 1731-260 is even dimmer ( $m \gtrsim 18$, Wijnands et al. $2001 \mathrm{~b}$ ), that makes our upper limit even stronger. The obtained upper limit on the surface temperature seems unreasonable taking into account the significant irradiation of the secondary star 
by the neutron star X-ray emission. Note here, that the size of the accretion disk usually has a radius comparable with the radius of the star, and the solid angle of the accretion disk, viewed from the neutron star surface is larger than that of the secondary. Illumination of the accretion disk by the X-ray source leads to its high brightness in the infrared spectral band (see e.g. Lyutyi \& Sunyaev 1976) which also strongly contradicts the existing infrared observations.

We can conclude that it is very unlikely that the observed 38 day periodicity in the X-ray light curve of KS 1731-260 is connected with the binary period of the system.

The presented consideration supports the assumption that KS 1731-260 is a low mass X-ray binary and we can anticipate that the infrared brightness of its possible counterpart should significantly decrease after the turnover of the X-ray source.

\subsection{Precession period?}

It is possible that the observed periodicity in the X-ray flux of KS 1731-260 is connected with the precession of the accretion disk, similarly to what is observed in the case of some other known X-ray binaries. Several X-ray binaries demonstrate precession of the accretion disk (e.g. Her X-1, Giacconi et al. 1973; SS433, Margon 1984; Cherepashchuk \& Yarikov 1991; GRO J1655-40, Hjellming \& Rupen 1995) etc. Note however, that in two latter cases the evidence for the disk precession is coming mainly from the precession of the relativistic jets.

Assuming that the detected X-ray period represents the precession period of the accretion disk we can roughly estimate the value of the system binary period. SS 433, Her X-1, LMC X-4 and SMC X-1 have the ratios of precession $P_{\mathrm{p}}$ and orbital $P_{\text {orb }}$ periods in the range $P_{\mathrm{p}} / P_{\text {orb }} \sim 12-22$. These values are consistent with the predictions of accretion disk precession theory (e.g. Larwood 1998). Using this ratio we could estimate the binary period $P_{\text {orb }} \sim 1-3$ days of KS 1731-260 and the dimensions of the system $\sim 1-2 R_{\odot}$.
Acknowledgements. Authors thank Marat Gilfanov, Eugene Churazov, Alexey Vikhlinin for useful discussions and Rudy Wijnands for useful remarks about the paper and for the important information about recent infrared observations of KS 1731-260. This research has made use of data obtained through the High Energy Astrophysics Science Archive Research Center Online Service, provided by the NASA/Goddard Space Flight Center.

\section{References}

Aleksandrovich, N., Revnivtsev, Aref'ev, et al. 2002, Astr. Lett., 28, 279

Barret, D., Motch, C., \& Predehl, P. 1998, A\&A, 329, 965

Barret, D., Mandrou, P., Roques, J. P., et al. 1993, A\&AS, 97, 241

Cherepashchuk, A., \& Yarikov, S. 1991, Soviet. Astron. Lett., 17, 258

Giacconi, R., Gursky, H., Jellog, E., et al. 1973, ApJ, 184, 227

Hjellming, R., \& Rupen, M. 1995, Nature, 375, 464

Larwood, J. 1998, MNRAS, 299, L32

Levine, A., Bradt, H., Cui, W., et al. 1996, ApJ, 469, L33

Lomb, N. R. 1976, Ap\&SS, 39, 447

Lyutyi, V., \& Sunyaev, R. 1976, SvA, 20, 290

Margon, B. 1984, ARA\&A, 22, 507

Muno, M., Fox, D., Morgan, E., et al. 2000, ApJ, 524, 1016

Narita, T., Grindlay, J., \& Barret, D. 2001, ApJ, 547, 420

Press, W., Teukolsky, S., Vetterling, W., \& Flannery, B. 1992, Numerical Recipes, 2nd ed. (Cambridge: Cambridge University Press), 569

Revnivtsev, M., \& Sunyaev, R. 2002, Astr. Lett., 28, 19

Scargle, J. D. 1982, ApJ, 263, 835

Smith, D. A., Morgan, E. H., \& Bradt, H. 1997, ApJ, 479, L137

Sunyaev, R. 1989, IAUC, 4839

Sunyaev, R., Gilfanov, M., Churazov, E., et al. 1990, Soviet. Astron. Lett., 16, 59

Warner, B. 1995, Cataclysmic variable stars (Cambridge, New York: Cambridge University Press)

Wijnands, R., Miller, J., Markwardt, C., et al. 2001a, ApJ, 560, L159

Wijnands, R., Groot, P., Miller, J., et al. 2001b, Astronomy Telegram 72 (http://atel.caltech.edu/)

Zombeck, M. V. 1990, Handbook of Astronomy and Astrophysics, Second Edition (Cambridge, UK: Cambridge University Press) 